\title{
A close look at fractional derivatives approximations
}

\author{
Ercília Sousa \\ CMUC, University of Coimbra \\ Portugal \\ Email: ecs@mat.uc.pt
}

\begin{abstract}
In this work we discuss the accuracy of some approximations for fractional derivatives. We analyze the cases in which the fractional derivatives are defined in a domain different from the real line and show how the order of accuracy can be affected by the presence of boundaries. We observe the accuracy can be easily lost, in particular near the boundary points, unless some conditions of regularity are satisfied or certain boundary conditions are verified.
\end{abstract}

\section{INTRODUCTION}

Different types of discretizations to approximate the fractional derivatives have been appearing in literature, specially first order discretizations and second order discretizations. Earlier approximations can be found in [6], [7], [11] and more recent works are for instance [1], [3], [4], [5], [13], [14], [15]. In these works different approaches are given on how we approximate the fractional derivative and as a starting point to solve fractional differential equations.

Some of the proofs regarding the order of accuracy of the approximations for the fractional derivatives assume we are dealing with functions defined in the whole real line. These proofs use tools, such as the Fourier transform, that can only be applied when we are considering the real line as the domain, and therefore the results obtained may not be easily generalized to domains which are not $\mathbb{R}$. When such discretizations are used to solve problems defined in other domains, namely a bounded interval $[a, b]$, some of the sufficient conditions imposed to reach a certain order of accuracy, in this case may not be enough to guaranty that accuracy. We present some examples to illustrate that we can easily loose the order of accuracy proved for the real line unless certain boundary conditions are verified. Additionally, we observe the lower order accuracy obtained it is many times associated with the points near the boundary.

We start to present an example with the oldest and most used first order approximation based in the Grünwald-Letnikov formula. In the third section, we discuss an approximation for the Riesz operator. For both cases, we show that when working with fractional derivatives, results holding in $\mathbb{R}$ may not hold in other domains.

In our examples we consider fractional derivatives of order $\alpha$, where $1<\alpha<2$, since many works are dealing with diffusive problems, which are modeled with the help of this fractional derivative. The left and right Riemann-Liouville derivative on the domain $a<x<b$, when $1<\alpha<2$, are defined respectively by [12]

$$
D_{a+}^{\alpha} u(x)=\frac{1}{\Gamma(2-\alpha)} \frac{d^{2}}{d x^{2}} \int_{a}^{x} u(\xi)(x-\xi)^{1-\alpha} d \xi
$$

and

$$
D_{b-}^{\alpha} u(x)=\frac{1}{\Gamma(2-\alpha)} \frac{d^{2}}{d x^{2}} \int_{x}^{b} u(\xi)(\xi-x)^{1-\alpha} d \xi .
$$

Note that here we can have $a=-\infty$ and $b=\infty$.

\section{THE GRÜNWALD-LETNIKOV APPROXIMATION}

We first consider a Grünwald-Letnikov type of approximation which is very well known in literature. This discretization was one of the first algorithms given to approximate the fractional derivative and it has been proved to be first order accurate under certain conditions. In general, it is assumed to be first order accurate, although, this is not always the case. The order of accuracy will depend on the regularity of the function we are considering and on its domain, namely, if the domain is or not the real line.

In this section we present some examples that illustrate how the accuracy can be easily lost. We start with theoretical results given in literature, which present sufficient conditions for an approximation, of the fractional derivative defined in $\mathbb{R}$, to be first order accurate.

Let us define the operator

$$
A_{h}^{p} u(x)=\frac{1}{\Gamma(-\alpha)} \frac{1}{h^{\alpha}} \sum_{k=0}^{\infty} \frac{\Gamma(k-\alpha)}{\Gamma(k+1)} u(x-(k-p) h),
$$

for $p \geq 0$ and where $h>0$ and $x \in \mathbb{R}$.

The next result assumes the operator $A_{h}^{p}$, for $p=0$, approximates the fractional derivative (1) and it presents sufficient conditions that guaranty this approximation is first order accurate. Since the proof uses Fourier transforms in space, the domain considered is $\mathbb{R}$ and the proof can not be generalized to other domains.

Theorem 1: [16] Let $\alpha$ be a positive number, $u \in$ $C^{[\alpha]+n+1}(\mathbb{R}), u \in L_{1}^{(r)}(\mathbb{R}), 0 \leq r \leq[\alpha]+n+2$ and consider the operator $A_{h}^{p}$ for $p=0, A_{h}^{0}$, defined by (3). Then the Riemman-Liouville derivative given by (1), for $a=-\infty$, verifies,

$$
D_{a+}^{\alpha} u(x)=A_{h}^{0} u(x)-\sum_{l=1}^{n-1}\left(a_{l} D_{a+}^{\alpha+l} u(x)\right) h^{l}+\mathcal{O}\left(h^{n}\right),
$$

uniformly in $x \in \mathbb{R}$, where the constants $a_{l}$ are independent of $h, u$ and $x$.

A similar proof appeared years later in [8] for the shifted approximation, that is, when we consider the operator $A_{h}^{p}$ for $p=1$ to approximate the fractional derivative. The theorem presented in [8] imposes less regularity conditions to $u$ than 
Theorem 1 presented in [16]. However, since the proof follows the same lines as the proof in [16], and additionally the same authors, presented a more general result in [9] where stronger regularity conditions for $u$ are used, we assume this latter version is the correct version. The result is the following.

Theorem 2 [9]: Let $1<\alpha<2, f \in C^{n+3}(\mathbb{R})$ such that all derivatives up to order $n+3$ belong to $L^{1}(\mathbb{R})$ and consider the operator $A_{h}^{p}$ defined by (3). Then the Riemman-Liouville derivative given by (1), for $a=-\infty$, verifies,

$$
D_{a+}^{\alpha} u(x)=A_{h}^{p} u(x)-\sum_{l=1}^{n-1}\left(a_{l} D_{a+}^{\alpha+l} u(x)\right) h^{l}+\mathcal{O}\left(h^{n}\right),
$$

uniformly in $x \in \mathbb{R}$, where the constants $a_{l}$ are independent of $h, u$ and $x$.

Theorem 1 and Theorem 2 do not assume the same conditions for the function $u$, although the proofs are similar. Theorem 1 says that for the approximation (3) to be first order accurate, it is sufficient to assume $u \in C^{(3)}(\mathbb{R})$, whereas Theorem 2 states that it is sufficient to assume $u \in C^{(4)}(\mathbb{R})$.

Some other results have been appearing in literature concerning the previous approximation (3). For instance, in [6] it is proved that such discretization is a first order approximation for the fractional derivative (1) defined with $a=0$. However the regularity conditions are stated in a broader sense, such as, assuming functions sufficiently smooth.

One of the first places we can find the first order discretization to the fractional derivative (1) is in [7]. There, we can also found some discussion on the accuracy, suggesting the first order accuracy. On page 141, one can read an interesting observation:

"In general, algorithms are imperfect. The relative error term will generally depend on $\alpha$ and $h$ (possibly on $x$ also, though not in the examples considered here) and should tend to zero as $h$ approaches zero."

We will see that the error can also depend on $x$, as suggested in this sentence.

Let us now consider the function $u$ defined in $[0, \infty)$ and not in $(-\infty, \infty)$. We can define the following mesh points

$$
x_{j}=j h, j=0,1 \ldots,
$$

where $h$ denotes the uniform space step. The shifted GrünwaldLetnikov formula, given by the operator $A_{h}^{p}$ for $p=1$, can be written as

$$
D_{G L, S}^{\alpha, h} u\left(x_{j}\right)=\frac{1}{h^{\alpha}} \sum_{k=0}^{j} \omega_{k}^{(\alpha)} u\left(x_{j+1-k}\right) .
$$

The coefficients $\omega_{k}^{(\alpha)}$ can be obtained by the recurrence formula

$$
\omega_{0}^{(\alpha)}=1, \quad \omega_{k+1}^{(\alpha)}=-\frac{(\alpha-k)}{k+1} \omega_{k}^{(\alpha)}, k \geq 0 .
$$

In Table I, Table II and Table III we show the order of accuracy of the discretization (4), when we consider fractional derivatives for two different functions $u(x)=x^{2}$ and $u(x)=$ $x^{3}$. These functions have derivatives of all orders for $x>0$. The error presented, in the tables, is defined by

$$
\max _{1 \leq j \leq N-1}\left|D_{G L, S}^{\alpha, h} u\left(x_{j}\right)-D_{a+}^{\alpha} u\left(x_{j}\right)\right| .
$$

We observe that in the case, presented in Table I, which considers $u(x)=x^{2}$, the first order accuracy is not verified but in the second case, presented in Table II, and regarding the function $u(x)=x^{3}$, we have first order convergence. Additionally the results, in Table I, suggest that the order of accuracy depends on $\alpha$ and we note the rate of convergence is of order $2-\alpha$.

\begin{tabular}{llll}
$\alpha=1.2$ & $\alpha=1.4$ & $\alpha=1.6$ & $\alpha=1.8$ \\
\hline $2.4669 \times 10^{-2}$ & $3.1002 \times 10^{-2}$ & $2.8361 \times 10^{-2}$ & $9.5907 \times 10^{-3}$ \\
$3.9098 \times 10^{-3}$ & $7.7874 \times 10^{-3}$ & $1.1290 \times 10^{-2}$ & $6.0513 \times 10^{-3}$ \\
0.79 & 0.6 & 0.4 & 0.19
\end{tabular}

TABLE I. ERROR DEFINED BY (5) WHEN $h=1 / N$ CHANGES FROM $1 / 60$ TO $1 / 600$, FOR THE DISCRETIZATION $D_{G L, S}^{\alpha, h} u$ WHEN $u(x)=x^{2}$.

\begin{tabular}{llll}
$\alpha=1.2$ & $\alpha=1.4$ & $\alpha=1.6$ & $\alpha=1.8$ \\
\hline $4.2559 \times 10^{-2}$ & $2.2455 \times 10^{-2}$ & $2.8361 \times 10^{-2}$ & $1.0884 \times 10^{-2}$ \\
$4.2908 \times 10^{-3}$ & $2.2532 \times 10^{-3}$ & $1.1290 \times 10^{-2}$ & $1.0890 \times 10^{-3}$ \\
0.99 & 0.99 & 0.99 & 0.99 \\
\hline
\end{tabular}

TABLE II. ERROR DEFINED BY (5) WHEN $h=1 / N$ CHANGES FROM $1 / 60$ TO $1 / 600$, FOR THE DISCRETIZATION $D_{G L, S}^{\alpha, h} u$ WHEN $u(x)=x^{3}$.

Moreover, a close look at the first case, presented in Table I, where $u(x)=x^{2}$, motivated us to present the results in Table III. In Table I, it is shown the first order rate of convergence is not reached, but if we look at what happens at an interior point, we get the result displayed in Table III. In this table we have considered $\alpha=1.8$, which according to Table I, should lead to a convergence order of approximately $2-\alpha=0.2$. However, we are getting second order convergence. This happens because the points near the boundary are the ones slower in convergence. To compute the error (5), we have used the exact value of the derivative at $x=0.5$ which is 1.896275756503799 .

\begin{tabular}{ll}
$D_{G L, S}^{\alpha, \Delta x} u(0.5)$ & error \\
\hline 1.897513129133984 & $1.2373 \times 10^{-3}$ \\
1.896401905356470 & $1.2614 \times 10^{-4}$ \\
\hline
\end{tabular}

TABLE III. ERROR DEFINED BY (5) WHEN $h=1 / N$ CHANGES FROM $1 / 60$ TO $1 / 600$, FOR THE DISCRETIZATION $D_{G L, S}^{\alpha, h} u$ FOR $u(0.5)$ WHEN $\alpha=1.8$ AND $u(x)=x^{2}$. NOTE THAT FOR THE FIRST LINE THE VALUE $x_{j}=0.5$ IS TAKEN AT THE INTERIOR NODE $j=31$ AND IN THE SECOND LINE IS TAKEN AT $j=310$.

One can ask the question: if we have a function $u \in$ $C^{\infty}((0, \infty))$ and the approximation used was proved to be first order accurate in $\mathbb{R}$, why do we not have first order convergence, when we consider an extension of the function 
$u$ which is defined in $(0, \infty)$ to $\mathbb{R}$ ? The extension of such function would be a function $\widetilde{u}$ defined in $\mathbb{R}$, where $\widetilde{u}$ would be 0 outside the domain $[0, \infty)$, that is, defined by

$$
\widetilde{u}(x)=\left\{\begin{array}{lll}
u(x) & \text { if } & x>0 \\
0 & \text { if } \quad x \leq 0 .
\end{array}\right.
$$

Note that we are now only concerned with the left fractional Riemann-Liouville derivative.

In the particular case, when we consider the extension of $u(x)=x^{2}$ to $\mathbb{R}$, we obtain,

$$
\widetilde{u_{2}}(x)=\left\{\begin{array}{lll}
x^{2} & \text { if } \quad x>0 \\
0 & \text { if } \quad x \leq 0
\end{array}\right.
$$

For this case, Theorem 1 or Theorem 2 do not guaranty the first order accuracy because the sufficient conditions of Theorem 1, are verified by $u \in C^{(3)}((0, \infty))$, but they are not verified by the function $\widetilde{u}$. The extension has no second order derivative continuous, that is, $\widetilde{u} \notin C^{(2)}(\mathbb{R})$. We have a discontinuity at the point $x=0$, for the second order derivative. is,

For the case, where we extend the function $u(x)=x^{3}$, that

$$
\widetilde{u_{3}}(x)=\left\{\begin{array}{lll}
x^{3} & \text { if } & x>0 \\
0 & \text { if } & x \leq 0
\end{array}\right.
$$

we get first order accuracy, although Theorem 1 still demands more regularity. Now the function $\widetilde{u_{3}}(x) \in C^{2}(\mathbb{R})$, but it has no third order derivative continuous.

Note also that when we impose to $\widetilde{u}$, defined by (6), the regularity condition $\widetilde{u} \in C^{(r)}(\mathbb{R})$, this implicitly imposes at the boundary, $x=0$, the conditions

$$
u^{(k)}(0)=0, \quad k=1,2, \ldots, r .
$$

Therefore, in a domain different from the real line, to achieve order of accuracy $h$, it is sufficient to have a function with enough regularity and that satisfies (7).

Next, we are going to show in more detail what is the analytical error for these particular cases. The explicit expression for the truncation error of $u_{m}(x)=x^{m}, m=0,1,2, \ldots$ for this type of approximation is given in [14].

Theorem 3: [14] Let $u_{m}(x)=x^{m}, \quad m=0,1,2, \ldots$ Then

$$
\begin{aligned}
D_{G L, S}^{\alpha, h} u_{0}(x)= & D_{0+}^{\alpha} u_{0}(x)+h x^{-1-\alpha} \frac{(-\alpha)(-\alpha+3)}{2 \Gamma(1-\alpha)} \\
& +\mathcal{O}\left(h^{2}\right), \\
D_{G L, S}^{\alpha, h} u_{m}(x)= & D_{0+}^{\alpha} u_{m}(x)+h x^{m-1-\alpha} \frac{(2-\alpha) \Gamma(m+1)}{2 \Gamma(m-\alpha)} \\
& +\mathcal{O}\left(h^{2}\right) .
\end{aligned}
$$

Using the previous result, we can write explicitly the truncation error of the fractional derivative when $u(x)=x^{2}$, that is,

$$
T_{2}\left(x_{j}\right)=h x_{j}^{1-\alpha}(2-\alpha) \frac{\Gamma(3)}{\Gamma(2-\alpha)}+O\left(h^{2}\right) .
$$

Note that for $\alpha=1$ and $\alpha=2$ it is clear that we get respectively order of accuracy $O(h)$ and $O\left(h^{2}\right)$. This is in agreement with the fact that (4) becomes the well known approximations

$$
D_{G L}^{1, h} u\left(x_{j}\right)=\frac{u\left(x_{j+1}\right)-u\left(x_{j}\right)}{h}
$$

and

$$
D_{G L}^{2, h} u\left(x_{j}\right)=\frac{u\left(x_{j+1}\right)-2 u\left(x_{j}\right)+u\left(x_{j-1}\right)}{h^{2}} .
$$

Now, when we have $1<\alpha<2$, why do we get order $h^{2-\alpha}$ ? Note that for this case the leading term of the truncation error verifies

$$
h^{2-\alpha} j^{1-\alpha}(2-\alpha) \frac{\Gamma(3)}{\Gamma(2-\alpha)},
$$

and since $j \geq 1$, then $j^{1-\alpha} \leq 1$ and we obtain

$$
h^{2-\alpha} j^{1-\alpha}(2-\alpha) \frac{\Gamma(3)}{\Gamma(2-\alpha)} \leq h^{2-\alpha}(2-\alpha) \frac{\Gamma(3)}{\Gamma(2-\alpha)} .
$$

Note also, that from (8) and (9) when we compare the same value $u\left(x_{j}\right)$ obtained through different values of $h$ we get the first order accuracy.

In the case of Table II, where we have $u(x)=x^{3}$ the truncation error is given by

$$
T_{3}\left(x_{j}\right)=h x_{j}^{2-\alpha}(2-\alpha) \frac{\Gamma(3)}{\Gamma(2-\alpha)}+O\left(h^{2}\right)
$$

and here we can easily conclude the order of convergence is $h$.

We have observed that for certain domains the order of accuracy of the discretization (4), that approximates the fractional derivative of order $1<\alpha<2$, can be $h^{2-\alpha}$.

\section{THE RIESZ APPROXIMATION}

We now turn to another example which consists of a second order approximation for the Riesz operator. The Riesz operator is defined, for $1<\alpha<2$, by

$D_{R}^{\alpha} u(x)=\frac{-1}{2 \cos (\alpha \pi / 2)} \frac{1}{\Gamma(2-\alpha)} \frac{d^{2}}{d x^{2}} \int_{-\infty}^{\infty}|x-\xi|^{1-\alpha} u(\xi) d \xi$.

This operator can also be written in terms of the Riemann Liouville derivatives as the combination of the left and right Riemann-Liouville derivatives (1)-(2), in the following manner,

$$
D_{R}^{\alpha} u(x)=-\frac{1}{2 \cos (\alpha \pi / 2)}\left(D_{a+}^{\alpha} u(x)+D_{b-}^{\alpha} u(x)\right) .
$$

In this case we have both derivatives, left and right, indirectly involved.

One of the second order approximations for the Riesz operator was introduced in [10] and given by

$$
D_{R}^{\alpha, h} u(x)=\frac{1}{h^{\alpha}} \sum_{k=-\infty}^{\infty} g_{k}^{(\alpha)} u(x-k h),
$$

where

$$
g_{k}^{(\alpha)}=\frac{(-1)^{k} \Gamma(\alpha+1)}{\Gamma(\alpha / 2-k+1) \Gamma(\alpha / 2+k+1)} .
$$


These coefficients can be obtained by the recurrence formulas

$$
\begin{aligned}
g_{0}^{(\alpha)} & =\frac{\Gamma(\alpha+1)}{\Gamma(\alpha / 2+1)^{2}}, \\
g_{k+1}^{(\alpha)} & =\left(1-\frac{\alpha+1}{\alpha / 2+k+1}\right) g_{k}^{(\alpha)}, \quad k \geq 0
\end{aligned}
$$

and

$$
g_{-k}^{(\alpha)}=g_{k}^{(\alpha)} .
$$

Note that for $\alpha=2$, the approximation (14) also becomes the well known second order discretization

$$
D_{R}^{2, h} u\left(x_{j}\right)=\frac{u\left(x_{j+1}\right)-2 u\left(x_{j}\right)+u\left(x_{j-1}\right)}{h^{2}} .
$$

The work that first presented the approximation (14) did not study the order of accuracy of the approximation. However, more recently in [2], this approximation was used to approximate a fractional diffusion equation, and it was proved to be second order accurate under some sufficient conditions. In the next theorem we give that result.

Theorem 4[2]: Let $u \in C^{5}(\mathbb{R})$ and all derivatives up to order five belong to $L_{1}(\mathbb{R})$ and

$$
D_{R}^{\alpha, h} u(x)=\frac{1}{h^{\alpha}} \sum_{k=-\infty}^{\infty} g_{k}^{(\alpha)} u(x-k h)
$$

be the fractional difference. Then

$$
D_{R}^{\alpha, h} u(x)=D_{R}^{\alpha} u(x)+O\left(h^{2}\right) .
$$

The proof of Theorem 4 follows closely the proofs of the Theorem 1 and Theorem 2 presented previously and that can be seen in [16] and [9] respectively.

In what follows, we describe some numerical tests in a bounded interval $[0,1]$ similarly to what we have done, in the previous section, for the first order accuracy case. Again, we will observe that sometimes we will not have second order accuracy.

\begin{tabular}{llll}
$\alpha=1.2$ & $\alpha=1.4$ & $\alpha=1.6$ & $\alpha=1.8$ \\
\hline $1.0946 \times 10^{-3}$ & $2.3075 \times 10^{-3}$ & $4.2873 \times 10^{-3}$ & $5.8832 \times 10^{-3}$ \\
$1.5058 \times 10^{-4}$ & $5.1590 \times 10^{-4}$ & $1.5568 \times 10^{-3}$ & $3.4968 \times 10^{-3}$ \\
0.86 & 0.65 & 0.44 & 0.23
\end{tabular}

TABLE IV. ERROR DEFINED BY (5) WHEN $h=1 / N$ CHANGES FROM $1 / 60$ TO $1 / 600$, FOR THE DISCRETIZATION OF THE RIESZ OPERATOR WHEN $u(x)=x^{2}(1-x)^{2}$.

\begin{tabular}{llll}
$\alpha=1.2$ & $\alpha=1.4$ & $\alpha=1.6$ & $\alpha=1.8$ \\
\hline $7.3776 \times 10^{-5}$ & $1.4512 \times 10^{-4}$ & $2.8793 \times 10^{-4}$ & $5.9566 \times 10^{-4}$ \\
$1.6982 \times 10^{-6}$ & $4.5400 \times 10^{-6}$ & $1.0664 \times 10^{-5}$ & $1.7517 \times 10^{-5}$ \\
1.64 & 1.50 & 1.43 & 1.53
\end{tabular}

TABLE V. ERROR DEFINED BY (5) WHEN $h=1 / N$ CHANGES FROM $1 / 60$ TO $1 / 600$, FOR THE DISCRETIZATION OF THE RIESZ OPERATOR WHEN $u(x)=x^{3}(1-x)^{3}$

\begin{tabular}{llll}
$\alpha=1.2$ & $\alpha=1.4$ & $\alpha=1.6$ & $\alpha=1.8$ \\
\hline $2.1769 \times 10^{-5}$ & $3.9194 \times 10^{-5}$ & $7.3521 \times 10^{-5}$ & $1.6899 \times 10^{-4}$ \\
$2.1777 \times 10^{-7}$ & $3.9213 \times 10^{-7}$ & $8.1210 \times 10^{-7}$ & $1.9978 \times 10^{-6}$ \\
1.99 & 1.99 & 1.95 & 1.93
\end{tabular}

TABLE VI. ERROR DEFINED BY (5) WHEN $h=1 / N$ CHANGES FROM $1 / 60$ TO $1 / 600$, FOR THE DISCRETIZATION OF THE RIESZ OPERATOR WHEN $u(x)=x^{4}(1-x)^{4}$.

In the next tables we display the error defined by (5). In Table IV we present the case $u(x)=x^{2}(1-x)^{2}$ and in Table $\mathrm{V}$ the case $u(x)=x^{3}(1-x)^{3}$. In both cases we do not have second order convergence. For the case in Table IV we have order of accuracy of $h^{2-\alpha}$ and for the example in Table V, we have order of accuracy $h^{3-\alpha}$. In Table VI, we consider the case $u(x)=x^{4}(1-x)^{4}$ and we finally observe order of accuracy $h^{2}$ independent of the $\alpha$ value.

Why the theorem does not guaranty that we can still have second order convergence when we restrict the fractional derivative to be defined in a bounded interval?

In order to extend the function $u$ that is defined in a bounded interval $[0,1]$, according to Theorem 3 we need to consider an extension $\tilde{u} \in C^{(5)}(\mathbb{R})$ and such that $\tilde{u}(x)=u(x)$ for $x \in[0,1]$ and zero otherwise. Additionally for the extension to be $C^{(5)}(\mathbb{R})$, we should have

$$
u^{(r)}(0)=0, \quad u^{(r)}(1)=0, \quad r=0,1,2,3,4,
$$

otherwise we will have a discontinuity at the boundary points, $x=0$ and $x=1$, for some of the derivatives.

The question can be formulated in this way: do we still have second order accuracy if one of the conditions (15) is not satisfied? For instance, for the case presented in Table IV, we can consider the extension

$$
\widetilde{u_{4}}(x)= \begin{cases}x^{2}(1-x)^{2} & \text { if } \quad 0<x<1 \\ 0 & \text { if } \quad x \leq 0, \quad x \geq 1 .\end{cases}
$$

However, this function is only in $C^{1}(\mathbb{R})$. For the case in Table $\mathrm{V}$, the respective extension would be given by

$$
\widetilde{u_{5}}(x)= \begin{cases}x^{2}(1-x)^{2} & \text { if } \quad 0<x<1 \\ 0 & \text { if } \quad x \leq 0, \quad x \geq 1\end{cases}
$$

and this function is in $C^{2}(\mathbb{R})$ but not in $C^{3}(\mathbb{R})$. For the case in Table VI, although the extension

$$
\widetilde{u_{6}}(x)= \begin{cases}x^{4}(1-x)^{4} & \text { if } \quad 0<x<1 \\ 0 & \text { if } \quad x \leq 0, \quad x \geq 1\end{cases}
$$

is only in $C^{3}(\mathbb{R})$ we get second order accuracy. This is not predicted by Theorem 3 , since the sufficient conditions of Theorem 3 are stronger. They only guarantee the convergence if we have a function in $C^{5}(\mathbb{R})$. These numerical tests are telling us that the sufficient conditions given in Theorem 3 are too strong for our particular case.

We have observed that for some particular cases the order of accuracy of the approximation (14), instead of being $O\left(h^{2}\right)$, it can be $O\left(h^{2-\alpha}\right)$ or $O\left(h^{3-\alpha}\right)$, depending on how smooth the functions are when extended to $\mathbb{R}$. When the domain is a bounded interval this will interfere with some boundary values 
of the function derivatives, which in our examples, are at the values $x=0$ and $x=1$.

\section{CONCLUSION}

We have shown some examples on how certain finite difference approximations for the fractional derivatives which are first order and second order accurate, can loose accuracy when the fractional derivatives are defined in a different domain from the real line, and for some of these examples a theoretical explanation was given. This type of test can be done to some other type of approximations to check its liability considering the rate of convergence, when the domain under consideration is different from the real line. We have observed the results strongly depend on the regularity of the functions considered and the $\alpha$ value. The regularity imposed on the functions defined in $\mathbb{R}$, when restricted to a bounded domain imply certain boundary conditions should be satisfied.

\section{ACKNOWLEDGMENT}

Research supported by CMUC and FCT (Portugal), through European program COMPETE/FEDER.

\section{REFERENCES}

[1] K. Adolfsson, M. Enelund, S. Larsson. Adaptative discretization of fractional order viscoelasticity using sparse time history. Comput. Methods Appl. Mech. Engrg. vol 193, pp. 4567-4590, 2004.

[2] C. Çelik, M. Duman. Crank-Nicolson method for the fractional diffusion equation with the Riesz fractional derivative. Journal of Computational Physics, vol. 231, pp. 1743-1750, 2012.

[3] K. Diethelm, N.J. Ford, A.D. Freed. Detailed error analysis for a fractional Adams method. Numerical Algorithms, vol. 36, pp. 31-52, 2004.

[4] C. Li, A. Chen, J. Ye. Numerical approaches to fractional calculus and fractional ordinary differential equations. Journal of Computational Physics, vol. 230, pp. 3352-3368, 2011.

[5] Y.M. Lin, C.J. Xu. Finite difference/spectral approximations for the timefractional diffusion equation. Journal of Computational Physics, vol. 225, pp. 1533-1552, 2007.

[6] Ch. Lubich. Discretized fractional calculus. SIAM J. Math. Anal. vol. 17, pp. 704719, 1984.

[7] K.B. Oldham, J. Spanier. The fractional calculus, Dover, 1974.

[8] M.M. Meerschaert, C. Tadjeran. Finite difference approximations for fractional advection-dispersion flow equations. Journal of Computational and Applied Mathematics, vol. 172, pp. 65-77, 2004.

[9] C. Tadjeran, M.M. Meerschaert, H.P. Scheffler, A second-order accurate numerical approximation for the fractional diffusion equation. Journal of Computational Physics, vol. 213, pp. 205-213, 2006.

[10] M. Ortigueira. Riesz potential operators and inverses via fractional centred derivatives. Int. J. Math. Sci., 2006.

[11] I. Podlubny Fractional differential equations, Academic Press, San Diego, 1999.

[12] S.G. Samko, A.A. Kilbas, O.I. Marchinev, Fractional integrals and derivatives, Gordon and Breach Science Publishers, 1987.

[13] S. Shen, F. Liu. Error analysis of an explicit finite difference approximation for the space fractional diffusion equation with insulated ends. ANZIAM Journal, vol. 46 (E), C871-C887, 2005.

[14] E. Sousa, How to approximate the fractional derivative of order $1<$ $\alpha<2$. Int. J. Bifurcat. Chaos Vol. 22 (4), pp. 1250075, 2012.

[15] W.Y. Tian, H. Zhou, W.H. Deng. A class of second order difference approximations for solving fractional diffusion equations. Mathematics of Computation, in press.

[16] V.K. Tuan, R. Gorenflo, Extrapolation to the limit for numerical fractional differentiation. Z. Agnew. Math. Mech. 75 pp. 646648, 1995. 